\title{
EFFECTIVE HEURISTIC ALGORITHM FOR DYNAMIC ROUTING AND BANDWIDTH MANAGEMENT UNDER QUALITY OF SERVICE CONSTRAINTS IN MULTISTAGE INTERCONNECTION NETWORKS
}

\author{
${ }^{1}$ Deepalakshmi, R. and ${ }^{2}$ S. Rajaram \\ ${ }^{1}$ Department of Computer Science and Engineering Velammal College of Engineering and Technology, \\ Madurai, Tamil Nadu, India \\ ${ }^{2}$ Department of Electronics and Communication Engineering, \\ Thiagarajar College of Engineering, Madurai, Tamil Nadu, India
}

Received 2013-11-08; Revised 2013-11-26; Accepted 2014-01-07

\begin{abstract}
Today, Internet of Things (IoT) has introduced abundant bandwidth consumption and necessities in multimedia communications from online games to video-conferencing applications with the constraint of QoS requirements from time to time. The expected rapid proliferation of services would require performance unprecedented in the currently available best-effort routing algorithms. In, particular, the present routing mechanisms are based on the best-effort paradigm are unlikely to provide satisfactory endto-end performance for services required in the real time applications. Thus, there is a definite need for architectures and algorithms that provide bandwidth guaranteed and QoS guarantees beyond those of the currently available ones. The proposed routing algorithm addressed the problem by computing low cost trees with delay bounded within the model wherein the bandwidth can be reserved and guaranteed once reserved on various links of the network there by providing QoS guarantees. This novel tree-pruning algorithm aids the bandwidth measurement tools by applying heuristic approach and the effectiveness of the algorithm is determined by two factors (i) the end-to-end delay (ii) the cost of routing. The new data structure significantly improves the running time complexity by $\mathrm{O}(\log \mathrm{k})$ for routing procedures under a variety of QoS constraints and checking tree routing runs in $\mathrm{O}\left(\mathrm{m}+\mathrm{n}^{2}\right)$.
\end{abstract}

Keywords: Quality of Service (QoS), Network Traffic, Bandwidth Allocation, Bandwidth Management, Wavelength Links, Multistage Networks

\section{INTRODUCTION}

Data flows belonging to multimedia applications are gaining importance in the Internet. A key characteristic of such data flows is that they require Quality of Service (QoS). An Internet populated with data flows requiring QoS constitutes a paradigm change from the Internet in its early days. This has been accounted for in many research endeavours proposing new architectures, algorithms and protocols. However, one area that has been relatively underexposed is the development of new models for QoS Zhenga and Mouftahb (2009). Hence, the vision that has inspired this dissertation is the development of a unified model for the performance analysis of QoS in the optical networks Helmy et al. (2012). The traffic generated by multimedia applications has different requirements than those of traditional data traffic. These requirements pertain to: (i) bandwidth-multimedia streams use bandwidth for long periods of time in a continuous fashion, while data traffic is bursty (ii) multipoint communications-multimedia streams are expected to make heavy use of multicasting, while data

Corresponding Author: Deepalakshmi, R., Department of Computer Science and Engineering Velammal College of Engineering and Technology, Madurai, Tamil Nadu, India 
traffic uses it only occasionally and (iii) low latency, required by interactive communications. Deng and Subramaniam (2005) in their study stated that the present optical network resource provisioning mechanisms are based on the best-effort paradigm is unlikely to provide satisfactory end-to-end performance for services required in the real time applications. Provisioning of a transport network refers to assigning network resources to a static traffic demand Munoz et al. (2009). The routing algorithms used in deployed networks are not able to take these new requirements into account they find routes from a topological point of view only studied by Martini et al. (2009) and Fouli et al. (2009). Thus, there is a definite need for architectures and algorithms that provide bandwidth guaranteed and QoS guarantees beyond those of the currently available ones. In this thesis, we devise a routing algorithm for multimedia streams that not only satisfies the requirements, but is efficient, i.e., optimizes the usage of network resources in order to maximize that amount of traffic it can carry.

Bandwidth of the optical network is not always efficiently utilized for data transmission because network traffic typically exhibits a high degree of burstiness. Song et al. (2010) proved that the bursty network traffic results in some transmission time slots that consistently overflow even under a very light traffic load. The result of Hwang et al. (2012) work showed that bursty network traffic results in some transmission time slots that are not completely filled even when the overall traffic load is very high. In view of the above concern, there is a need for an access network based on Optical technology that efficiently utilizes the available bandwidth by reducing the amount of band width wasted because transmission time slots are not filled to the maximum capacity. In the proposed work the algorithm uses an interleaved polling scheme to efficiently use the available bandwidth of the network.

Bhatia et al. (2008) investigated the problem of reliable multi-path provisioning for high-capacity backbone optical networks it proposed and investigated the characteristics of effective multi-path bandwidth as a metric to provision a connection to multiple paths while satisfying its availability requirements. Morea et al. (2008) proposed an algorithm the results showed that the heuristics perform significantly better in decreasing blocking probability than conventional single-path provisioning approaches for high bandwidth connections. Further the problem of minimizing the rejection ratio of provisioning algorithms when (1) the residual bandwidths on links of the network backbone are finite and (2) multiple cross connects need to be established on-line on the network backbone Wu et al. (2011). One of the requisites of a good provisioning algorithm is that it should achieve a low rejection ratio. However, previous provisioning algorithms proposed by Tibuleac and Filer (2010) and Gringeri et al. (2010) have been unable to meet this requirement. This raises the research questions of how to design an adaptive algorithm to distribute the traffic onto the available multiple paths and how to allocate the bandwidth resource to protection path to satisfy the QoS under a dynamic traffic requests. This chapter therefore proposes a new provisioning algorithm, a Dynamic Cost Optimized Provisioning Algorithm (DCOPA) to address these issues.

In this study the main idea is to grouping similar links having equivalent available bandwidth of consecutive time slots with constraint to form larger time slots. This process can be done until there is only one time interval left which is the maximum time interval to be considered Siamak Azodolmolkya et al. (2009). This type of grouping allows us to find the minimum bandwidth in network topology during any time interval in a fast way and have fairness in under-utilizationcircumstances. We introduce a new algorithm named optimal tree to record this. It is assumed that each connection requires a dedicated wavelength on each link of its path. We focus on tree based provisioning strategies for optical networks. We consider the formulation of the primary network design as a graphical representation to present binary search tree heuristic in our approach. Time variant dynamic nature of available bandwidth is estimated via measurement tools such as pathchar, pathload,pathneck and methods like probing packet trains, packet train gap model, Self Loading Periodic Streams (SLoPS), statistical processing, kalman filtering.We use these measurements for bandwidth provisioning. After the measurements values are calculated in the network design, it is reserved via link multiplexing technique using our optimal tree algorithm and binary search tree concept to be used to provisioning bandwidth Deepalakshmi et al. $(2010 ; 2011)$.

This study is thus organized as follows: Section 2 gives a brief scenario of new bandwidth reservation model. Section 3 develops the routing algorithms for routing based on QoS requirement and simulation results of the traffic performance in term of blocking probability, end-to-end delay versus the traffic loads. Finally conclusions and some aspects of future works are given. 


\section{NEW BANDWIDTH RESERVATION MODEL}

Each link in a network has a bandwidth available during a given time slot. Divide the time axis into slots and each slot represents the minimal time unit use by service requirements. The bandwidth status for each link can be given in an array. When there is a service requirement to be satisfied, the bandwidth status is updated.

An optimal tree with $\mathrm{k}$ nodes, vertex $\mathrm{v} \in \mathrm{V}$ keeps the information like $I_{1}(v), I_{2}(v)$ the left and right end of the interval covered by $\mathrm{v}$, the pruned bandwidth information $\mathrm{I}_{1}(\mathrm{v}), \mathrm{I}_{2}(\mathrm{v})$ accumulate minimal bandwidth in node $\mathrm{v}$.the interval in node $\mathrm{v}$ consists of intervals in the child nodes. There is a minimal bandwidth on each interval for the value for a node $\mathrm{v}$, if the node $\mathrm{v}$ is the root of the optimal tree and then the minimum bandwidth on time interval [1 $(\mathrm{v}), \mathrm{r}(\mathrm{v})]$. In general the path from the root to $\mathrm{v}$ in the bandwidth tree is $\left(\mathrm{v}_{1}, \mathrm{v}_{2}, \ldots, \mathrm{v}_{\mathrm{k}}\right)$ where $1 \mathrm{v}$ is the root an $\mathrm{v}_{\mathrm{k}}-$ $\mathrm{v}$ then $\sum_{\mathrm{i}-1}^{\mathrm{K}}$ bulid_up(v).

Figure 1 and 2 illustrate a bandwidth distribution example and corresponding bandwidth tree.

\subsection{Minimum Bandwidth on a Path}

A recursive algorithm for finding the minimum bandwidth for a given time interval is shown in Fig. 3, in which $v_{1}$ is the originator node of optimal tree and $\mathrm{v}_{2}, \mathrm{v}_{3}$ are the left and right subtree of the interval of the service requirement respectively it is not difficult to see that if $\left(\mathrm{v}_{1}, \mathrm{v}_{2}, . ., \mathrm{v}_{\mathrm{k}}\right)$ is the path from the root $\mathrm{v}_{1}$ to the node $\mathrm{v}_{\mathrm{k}}$.

Then the value $\mathrm{v}_{\mathrm{b}}$ returned by the algorithm $\min _{\mathrm{BW}}$ on node $\mathrm{v}$ makes $\mathrm{v}_{\mathrm{b}}+$ build_up $\left(\mathrm{v}_{1}\right)+\ldots+$ build_up $\left(\mathrm{v}_{\mathrm{k}-1}\right)$ to equal the minimum bandwidth on the interval $\left[\mathrm{v}_{\mathrm{i}}, \mathrm{v}_{\mathrm{i}+1}\right]$. In particular, if $\mathrm{v}$ is the root of an optimal tree, the value $\mathrm{v}_{\mathrm{b}}$ is the minimum bandwidth on the interval $\left[\mathrm{v}_{\mathrm{i}}, \mathrm{v}_{\mathrm{i}+1}\right]$.

\subsection{Bandwidth Allocation and Release on a Path}

Now we consider how to update the bandwidth tree if we want to allocate bandwidth to a service requirement. Algorithm alloc ${ }_{B W}$ in Fig. 4 updates the bandwidth optimal tree when some bandwidth resource is allocated to a service requirement. In alloc ${ }_{\mathrm{BW}}, \mathrm{r}_{0}$ is the root node of the bandwidth optimal tree, $w$ is the bandwidth requirement and $[i, j]$ is the time interval of the service requirement. The algorithm will check whether the bandwidth request can be satisfied by calling function $\min _{\mathrm{BW}}$ to compute the minimum bandwidth that can be supported during time interval $[\mathrm{i}, \mathrm{j}]$ on the link.

If minimum bandwidth is lower than $\mathrm{w}$, than the function just return. If the bandwidth requirement can be satisfied, alloc ${ }_{\mathrm{BW}}$ calls the function spilt () to allocate bandwidth to the bandwidth requirement and the function merge () to merge nodes if necessary.

\subsection{Checking Feasibility of a Connection}

In the basic reservation model, a service request has a source node 's' a destination node ' $d$ '. The starting time of the service is $t_{s}$ and the duration of it is $\tau$. The bandwidth requirement of the service is $\beta$. We need to solve the following problems.

\subsection{Connection Feasibility}

In this case, given the starting time of the service on the duration of it and the algorithm need to find a path in the network that can satisfy the requirement if there is afeasible path. Given a connection request with source node 's', destination node ' $d$ ', bandwidth $\beta$, starting time $t_{s}$ and duration $\tau$ find a path $p$ that satisfies the bandwidth requirement during the time interval $\left[\mathrm{t}_{\mathrm{s}}, \mathrm{t}_{\mathrm{s}}+\tau\right]$. This problem can be solved easily because we already have the algorithm to check minimum bandwidth on a link during a given time interval. The flowing algorithm in Fig. 5 shows the connection feasible problem. A request $\left(\beta, t_{1}, t_{2}\right)$ denotes a service request with bandwidth requirement $\beta$, starting time $t_{1}$ and ending time $t_{2}$. If $t_{2}$ is $+\alpha$, then the request need to be satisfied as long as possible.

To find a path that can satisfy the service requirement as long as possible the time that each link can satisfy the service requirement. Create a time length graph $G^{\prime}$ on which each link has weight as the time length the link can satisfy the service requirement. The claim that is to find the maximum spanning tree in the new graph, then the path between source node sand destination node $d$ will be the path that can support the service requirement with themaximum duration.

\subsection{Checking Delay Constraints}

For many applications, bandwidth requirement and delay constraint both need to be satisfied. The delay along a path can be bounded by the summation of the delays on the links consisting of the path. The delay along a path is bounded differently depending on which service model we use. 


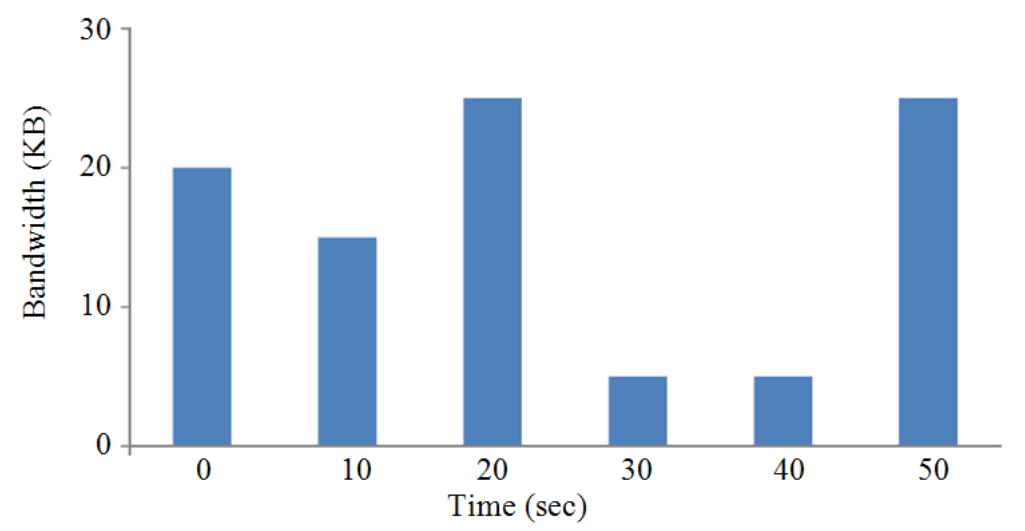

Fig. 1. Bandwidth distribution example

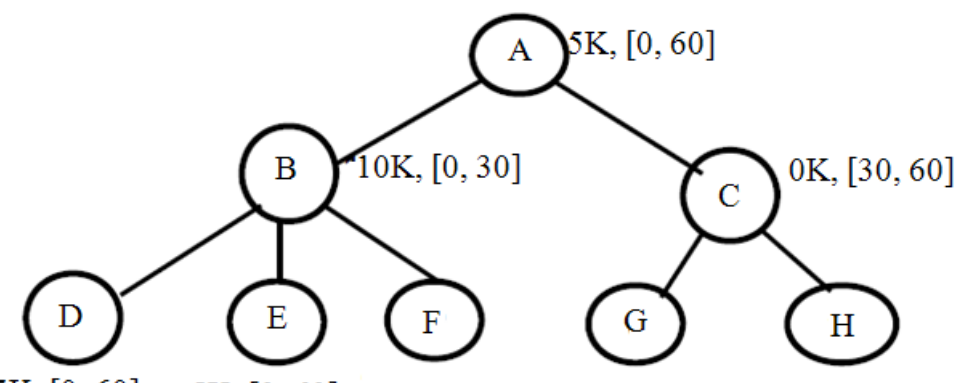

$5 \mathrm{~K},[0,60] \quad 5 \mathrm{~K},[0,60] \quad 10 \mathrm{~K},[20,30] \quad 0 \mathrm{~K},[30,50] \quad 20 \mathrm{~K},[50,60]$

Fig. 2. Bandwidth reservation on optimal tree

Algorithm. $\min _{B W}\left(v_{1}, v_{i}, v_{i+1}\right)$

Input: A node $\mathrm{v}$ in a bandwidth tree and time interval $\left[v_{i}, v_{i+1}\right]$

Output: The minimum bandwidth of the interval $\left[v_{i}, v_{i+1}\right]$.

If interval $\left[v_{i}, v_{i+1}\right]$ is not totally included in $[l(v), r(v)]$ then

Return 0;

If $\mathrm{v}$ is a leaf node or $[l(v), r(v)]=\left[v_{i}, v_{i+1}\right]$ then

Return build_up $(v)$;

$\min _{B W}=+\infty$;

For each child node $u$ of $\mathrm{v}$ such that $\left[v_{i}, v_{i+1}\right] \cap[l(v), r(v)] \neq \phi$

Do

Value $=\min _{B W}(\mathrm{u}, \max (\mathrm{l}(\mathrm{u}), \mathrm{c}), \min (\mathrm{r}(\mathrm{u}), \mathrm{d}))$;

If $\min _{B W}>$ value then

$\min _{B W}=$ value;

Return build_up(v)+ $\min _{B W}$;

Fig. 3. Algorithm to reserve minimum bandwidth 
Deepalakshmi, R. and S. Rajaram / American Journal of Applied Sciences 11 (3): 414-424, 2014

\section{Algorithm.}

Input: a bandwidth tree rooted at , bandwidth requirement $\mathrm{w}$ and time interval

Output: an updated bandwidth tree.

$=$

if $\operatorname{minBW}<\mathrm{w}$ then

return;

split;

if count $>1$ then

create new root node ;

Set $\quad, \ldots, \quad$ to be the children of ;

Set the accumulate bandwidth on

$=$;

merge ;

merge ;

return ;

Fig. 4. Algorithm for bandwidth allocation on a link

Algorithm. normalize

normalize $(u)$

$1(u)=1 \quad$ (u's left most child);

$r(u)=r \quad$ (u's right most child $)$;

$\min _{B W}=$ the minimum accumulate bandwidth among u's children;

build_up $(u)=$ build_up $(u)+\min _{B W}$;

for u's each child $v$ do

build_up $(v)=$ build_up(v)- $\min _{B W}$;

return;

Fig. 5. Algorithm normalize 
We consider the rate-base service model is used in our research work. In this model, the delay along a path can be bounded by the following formula:

$$
\mathrm{D}(\mathrm{p}, \mathrm{r})=(\sigma+\mathrm{n}(\mathrm{p}) * \mathrm{c}) / \mathrm{r}+\sum_{1} \delta_{1}
$$

Where:

$\mathrm{p} \quad=$ The path

$\mathrm{n}(\mathrm{p})=$ The number of hops on path $\mathrm{p}$

$r \quad=$ The allocate bandwidth

$\sigma=$ The burst size of the connection

$\mathrm{C}=$ The maximal packet size and is the constant delay on link 1

The problem of finding a path to satisfy both bandwidth requirement and delay constrain is finally described as the following.

\subsection{Bandwidth and Delay Problem}

Given a connection request with source node $s$ an destination node $\mathrm{d}$, bandwidth requirement $\mathrm{B}$, delay constraint $D_{0}$, starting time $t_{s}$ an duration $\tau$ find a path $p$ from $\mathrm{s}$ to $\mathrm{d}$ that can support the bandwidth and delay constraints during time $\left[\mathrm{t}_{\mathrm{s}}, \mathrm{t}_{\mathrm{s}}+\tau\right]$.

\section{PRACTICAL AND THEORETICAL ASPECTS OF ROUTING RESULTS}

After being able to build and check tree pruning algorithm in an arbitrary network $\mathrm{G}$, let us discuss some interesting results and find some surprising practical as well as important theoretical consequences when comparing different routing approaches.

\subsection{Optimal Vs Suboptimal Tree}

The differences between an optimal and suboptimal tree shown in Fig. 6 which illustrates how the overall cost of an optimal tree routed may be considerably less than the cost of suboptimal tree in a Dijkstra shortest path suboptimal tree model for a network G. That may be achieved by comparing them to an absolute value, i.e., the cost in $G$ when routing a single traffic matrix $M$. $M$ is coptimal treen from all possible traffic matrices that make maximal use of all $b^{-}$and $b^{+}$. This means that for $T_{m}=(u, v)$ Equation (1):

$\sum_{\mathrm{u} \in \mathrm{Q}} \mathrm{m}_{\mathrm{u}, \mathrm{v}} \leq \mathrm{b}_{\mathrm{v}}^{-}$and $\sum_{\mathrm{v} \in \mathrm{Q}} \mathrm{m}_{\mathrm{u}, \mathrm{v}} \leq \mathrm{b}_{\mathrm{u}}^{+}$

This problem of finding $\mathbf{M}$ constrained by above notation may be solved as a maximum flow problem between all $\mathrm{u}$ and $\mathrm{v}$ depicted in Fig. 6. Since we assume no traffic from a node $v$ to itself, there is no connection between $\mathrm{v}$ on the left to $\mathrm{v}$ on the right side of a graph.

In the following, the simulation results for the two models compared to the minimally required cost by $\mathrm{M}$, first with symmetric bound $b_{v}^{-}=b_{v}^{+}$for all $v \in Q$, then with asymmetric bounds $b_{v}^{-} \neq b_{v}^{+}$. The data of every simulation is based on 20,000 generated graphs $\mathrm{G}$ with for every possible configuration, there were 100 random variants optimal tree. Thus, there were for example 100 graphs with $\mathrm{n}=3, \mathrm{~m}=2$ and $\mathrm{q}=3$ and 100 graphs with $\mathrm{n}$ $=10, \mathrm{~m}=45$ and $\mathrm{q}=10$.

\subsection{Comparing Tree, Prunable Reservations}

Defined $\mathrm{W}=\sum_{\mathrm{e} \in \mathrm{E}} \mathrm{c}_{\mathrm{e}} \cdot \mathrm{y}_{\mathrm{e}}$ as the overall weight or cost of any optimal tree reservation in a graph $\mathrm{G}$. Let now $\mathrm{C}_{\mathrm{T}}$ be the cost of a tree reservation in $G$ and $C_{S}$ the cost for a splittable reservation. Obviously, the following comparison is always valid: $C_{T} \geq C_{S}$ Even though the graph in Fig. 6 has only three nodes, finding the given splittable reservation routing is not trivial and thus list it here.

The result is approximately the same for networks with 3 to 5 nodes. Additional experiments show the same ratio when $G$ has 6 or 7 nodes. Interestingly, there were onlytwo cases in all examples where tree routing found a better routing than splittable tree routing. Here, however, network size might play a role. Let us now look at simulation results to find some interesting facts about asymmetric tree, splittable routing. Once again, we work with randomly generated networks. They include 6200 graphs with 3 to 5 nodes, formally described in Table 1.

Bounds $b$ are chosen less strictly this allows for a wider range of solutions, especially when comparing tree to splittable routing reservations where b-values have a strong influence on routing paths.

In Fig. 7 we show that for every fifth network $G$ that we look at, splittable routing reserves less bandwidth than tree routing. The result is approximately the same for networks with 3 to 5 nodes. Additional experiments show the same ratio when $G$ has 6 or 7 nodes. Interestingly, there were only two cases in all examples where tree routing found a better routing than splittable tree routing. Here, however, network size might play a role.

\subsection{Comparing Success Ratio, Average Message Overhead, Average Message Cost}

Extensive simulations were done to evaluate the proposed tree-pruning algorithm. Three performance metrics, success ratio, average message overhead and average path cost, are defined as follows: 
Success ratio $=\frac{\text { Number of connections accepted }}{\text { Total number of connections requested }}$

Average message overhead $=$

Total number of routing messages sent

Total number of connections requested

Average path cost $=$

$\underline{\text { Total cost of all established connection paths }}$

Number of established connections paths

\subsection{Two Algorithms are Simulated}

The optimal tree algorithm and BST. Each routing packets accrues the delay on the path it has traversed and the packets proceeds only if the accumulated delay does not exceed the delay bound. When certain scheduling policies are used and the routing packets are not set to the appropriate priority, the routing packets travel at speeds according to the link delays. Hence, the packets traveling along the least delay path arrives first. An intermediate node needs only to propagate the first receive packet and discard all successively received ones. There will be at most one packet sent along every link. The algorithm fins feasible path whenever there exists one and hence is the optimal algorithm in terms of success ratio. The BST algorithm does not have an efficient mechanism for the termination detection. It selects the routing path when the destination receives the first routed packet. The advantage of the BST algorithm is that it does not need to maintain any global state. The disadvantage is that too many routing packets are sent.

Table 1. Simulation result splittable routing

\begin{tabular}{lll}
\hline$(\mathrm{u}, \mathrm{v})$ & Splittable & \\
\hline $\mathrm{q} 1 \rightarrow \mathrm{q} 2$ & $50 \%: \mathrm{q} 1, \mathrm{q} 2$ & $50 \%: \mathrm{q} 1, \mathrm{q} 3, \mathrm{q} 2$ \\
$\mathrm{q} 1 \rightarrow \mathrm{q} 3$ & $50 \%: \mathrm{q} 1, \mathrm{q} 3$ & $50 \%: \mathrm{q} 1, \mathrm{q} 2, \mathrm{q} 3$ \\
$\mathrm{q} 2 \rightarrow \mathrm{q} 1$ & $50 \%: \mathrm{q} 2, \mathrm{q} 1$ & $50 \%: \mathrm{q} 2, \mathrm{q} 3, \mathrm{q} 1$ \\
$\mathrm{q} 2 \rightarrow \mathrm{q} 3$ & $50 \%: \mathrm{q} 2, \mathrm{q} 3$ & $50 \%: \mathrm{q} 2, \mathrm{q} 1, \mathrm{q} 3$ \\
$\mathrm{q} 3 \rightarrow \mathrm{q} 1$ & $50 \%: \mathrm{q} 3, \mathrm{q} 1$ & $50 \%: \mathrm{q} 3, \mathrm{q} 2, \mathrm{q} 1$ \\
$\mathrm{q} 3 \rightarrow \mathrm{q} 2$ & $50 \%: \mathrm{q} 3, \mathrm{q} 2$ & $50 \%: \mathrm{q} 3, \mathrm{q} 1, \mathrm{q} 2$ \\
\hline
\end{tabular}

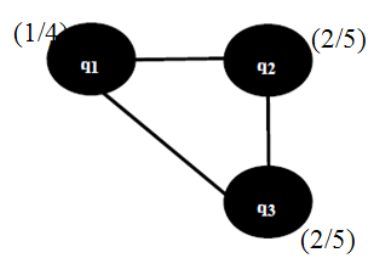

(a) Graph G

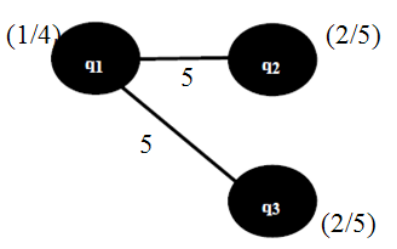

(b) Tree routing

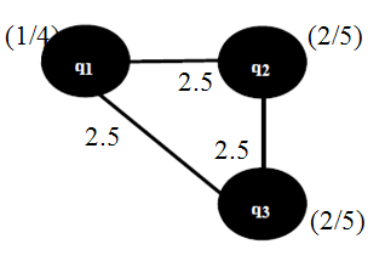

(c) Splittable routing

Fig. 6. For a network $\mathrm{G}$ in (a), tree routing in (b) has a cost of 10 while splittable routing in (c) has one of only 7.5

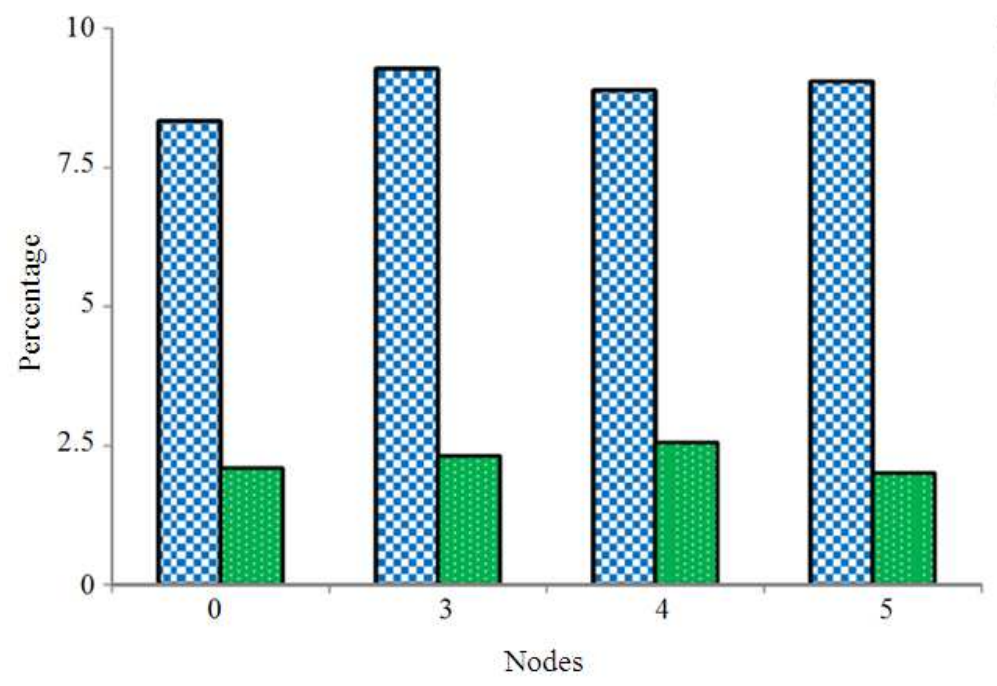

vTree routing

口Splittable rounting

Fig. 7. Percentage of networks $G$ that have a smaller reservation for splittable routing than for tree routing 


\subsection{Success Ratio}

Figure 8 and 9 compares the success ratios of the two algorithms. The success ratio is a function of the both average delay requirement $R_{d} D$ and imprecision ratio $\Gamma$. The former is represented by the $\mathrm{x}$ axis and the later is shown by different figure. In each figure, as the delay requirement become larger, it becomes easier to be satisfied and thus the success ratio is higher. The optimal tree algorithm, as expected, has the best success ratio. BST algorithm performs much worse when the imprecision ratio is $50 \%$. This is because BST searches multiple paths and the number of paths searched is adjusted according to how hard it will be to find a feasible path.

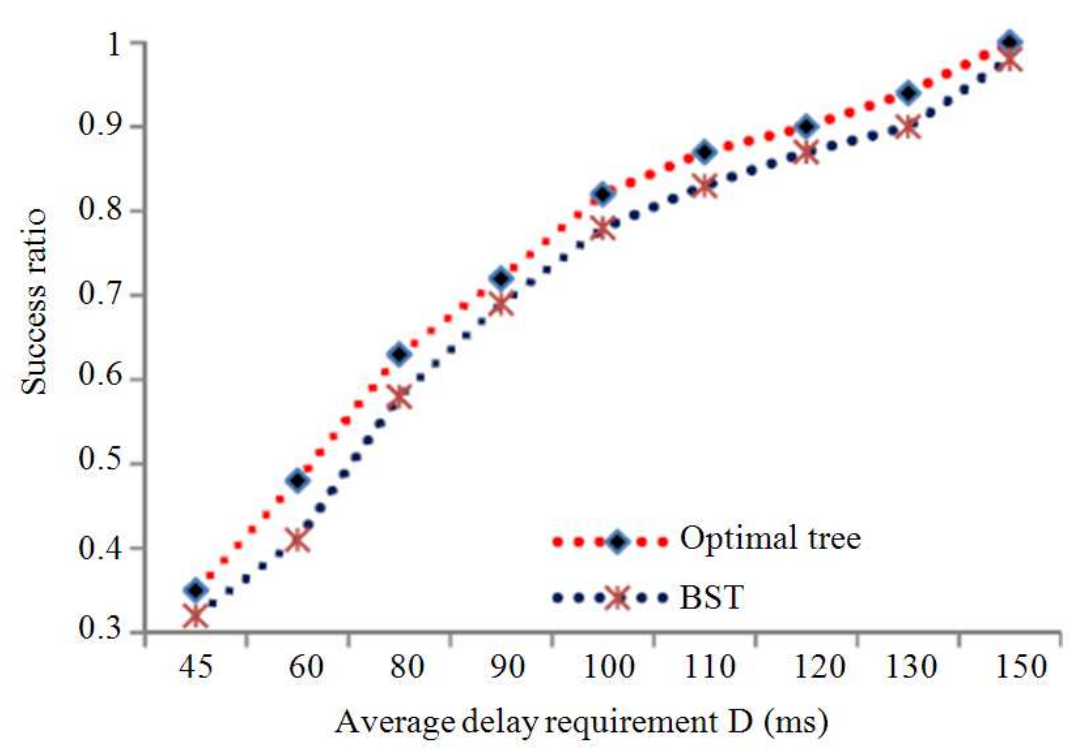

Fig. 8. Success ratio rate imprecision rate: $10 \%$

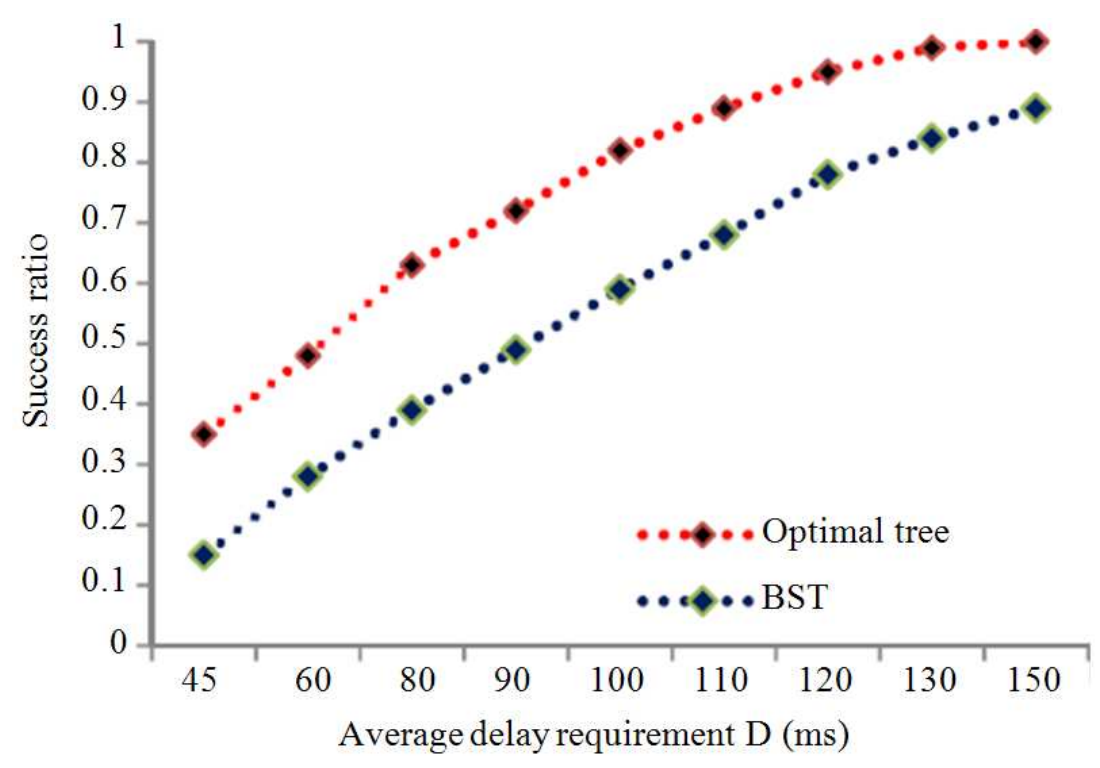

Fig. 9. Success ratio imprecision rate: $50 \%$ 


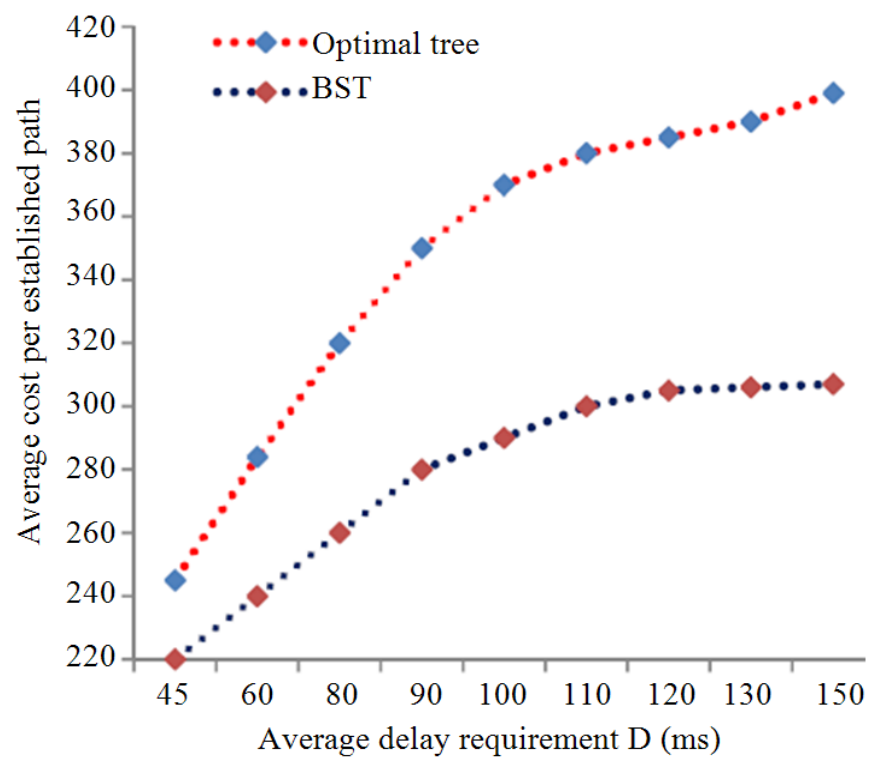

Fig. 10. Cost per established path imprecision rate: $10 \%$

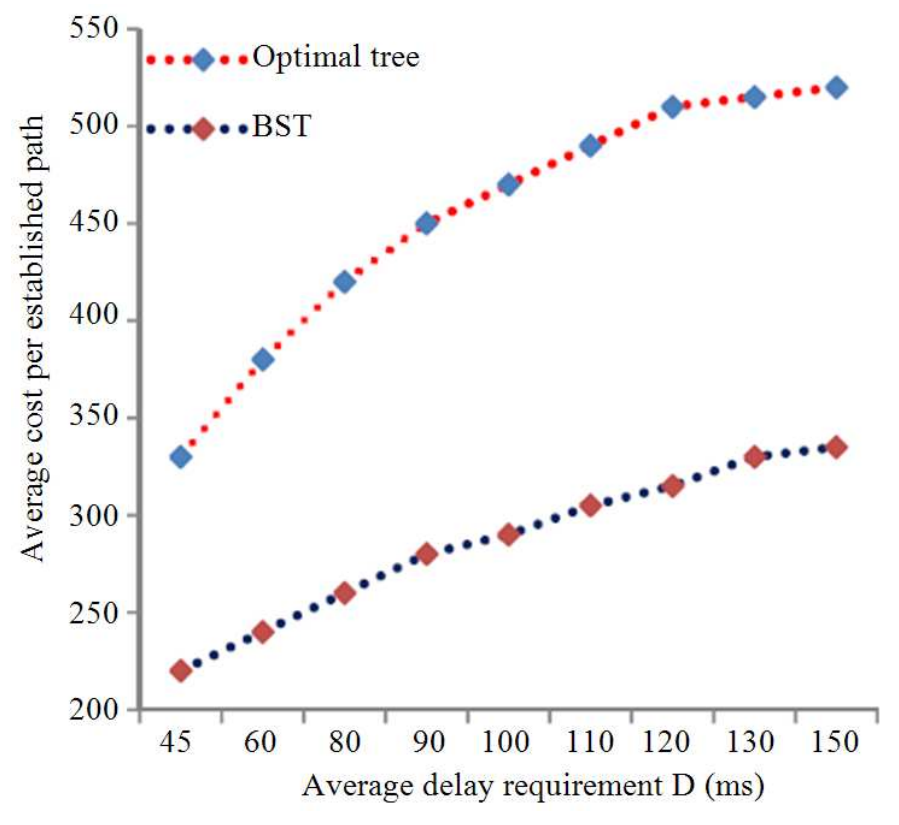

Fig. 11. Cost per established path imprecision rate: $50 \%$

In addition, the state information of intermediate nodes is collectively used to direct the probes along the most appropriate paths towards the destination. In contrast, the BST algorithm performs much worse when the imprecision rate is high.

\subsection{Average Path Cost}

In Fig. 10 BST has much lower path cost than the optimal tree algorithm. This is because BST uses both the delay metric an cost metric to make the routing decision while the other algorithm use only the delay 
metric. In Fig. 11 however, the average path cost of BST ishigher than that of optimal tree when D is relatively low. Optimal tree has a much higher success ration than BST when the imprecision rate is 50\%. Those connections, that optimal tree is able to establish but BST is not able to, tent to have relatively long routing paths, as observe in the simulation. They also tend to have higher cost, which brings the average path cost up. A fair comparison is made where only the connections which can be established by BST are considered. The average path cost of optimal tree is lower than that of BST.

\section{CONCLUSION}

We formulated two generic routing problems within the algorithm wherein the bandwidth can be reserve and guarantee once reserved on various links. The proposed new Heuristic Algorithm which records the interval of available link bandwidth instead of each individual time slot. The storage space and searching time complexity under this data structures are independent of the time slots. This overcomes the difficulty in the previous proposed research in which larger time slot size loses routing accuracy while smaller time slot size significantly increase routing complexity. Simulation experiments are done to evaluate the effectiveness of optimal tree under single class of service and multiple classes of service. The results show that optimal tree has better performance compared with other routing algorithms. Besides, the results indicate that optimal tree can properly provision differentiated service in terms of time complexity in a multiple service network environment.

By careful control the algorithm can achieve $100 \%$ throughput for uniform traffic. When the traffic is nonuniform, the algorithm quickly adapts to an efficient heap based policy among the busy queues. High performance is gained by integrating send/receive transmission in the order. Many important problems still remains open in the area of scheduling algorithms for real time traffic and data structures. It remains open to develop an efficient switching algorithm for multimedia traffic with complexity matching the best known switching algorithm. Switching the multimedia traffic using the above algorithms also still have to be developed.

\section{REFERENCES}

Azodolmolkya, S., M. Klinkowski, E. Marina, D. Careglio and J.S. Pareta et al., 2009. A survey on physical layer impairments aware routing and wavelength assignment algorithms in optical networks. Comput. Netw., 53: 926-944. DOI: 10.1016/j.comnet.2008.11.014
Bhatia, R.S., K. Murali, T.V. Lakshman and S. Sengupta, 2008. Bandwidth guaranteed routing with fast restoration against link and node failures. IEEE/ACM Trans. Network., 16: 1321-1330. DOI: 10.1109/TNET.2008.919325

Deepalakshmi, R., A. Divya and S. Rajaram, 2010. Multimedia traffic routing algorithm for optical networks with enhanced performance characteristics. Proceedings of the 4th International Conference on Internet Multimedia Services Architecture and Application, Dec. 15-17, IEEE Xplore Press, Bangalore, pp: 1-6. DOI: 10.1109/IMSAA.2010.5729406

Deepalakshmi, R., C.S. Kumar and S. Rajaram, 2011. New MAC protocol for traffic routing in optical networks by exploiting delays in dynamic bandwidth allocation. Proceedings of the IEEE International Conference on Computer Applications and Industrial Electronics, Dec. 4-7, IEEE Xplore Press, Penang, pp: 325-330. DOI: 10.1109/ICCAIE.2011.6162154

Deng, T. and S.S. Subramaniam, 2005. Adaptive QoS routing in dynamic wavelength-routed optical networks. Proceedings of the 2nd International Conference on Broadband Networks, Oct. 3-7, IEEE Xplore Press, pp: 184-193. DOI: 10.1109/ICBN.2005.1589614

Fouli, K., T. Berisa and M. Maier, 2009. Optical coding for enhanced real-time dynamic bandwidth allocation in passive optical networks. J. Lightwave Technol., 27: 5376-5384. DOI: 10.1109/JLT.2009.2031161

Gringeri, S., B. Basch, V. Shukla, R. Egorov and T.J. Xia, 2010. Flexible architectures for optical transport nodes and networks. IEEE Commun. Mag., 48: 40-50. DOI: 10.1109/MCOM.2010.5496877

Helmy, A., H. Fathallah and H. Mouftah, 2012. Interleaved polling versus multi-thread polling for bandwidth allocation in long-reach PONs. J. Opt. Commun. Network., 4: 210-218. DOI: 10.1364/JOCN.4.000210

Hwang, I.S., J.Y. Lee, K.R. Lai and A.T. Liem, 2012. Generic QoS-aware interleaved dynamic bandwidth allocation in scalable EPONs. J. Opt. Commun. Network., 4: 99-107. DOI: 10.1364/JOCN.4.000099

Martini, B., V. Martini, F. Baroncelli, K. Torkman and P. Castoldi, 2009. Application-driven control of network resources in multiservice optical networks. J. Opt. Commun. Network., 1: A270-A283. DOI: 10.1364/JOCN.1.00A270 
Morea, A., N. Brogard, F. Leplingard, J.C. Antona and T. Zami et al., 2008. QoT function and $\mathrm{A}^{\wedge} *$ routing: An optimized combination for connection search in translucent networks. J. Opt. Netw., 7: 42-61. DOI:10.1364/JON.7.000042

Munoz, R., R. Martinez and R. Casellas, 2009. Challenges for GMPLS lightpath provisioning in transparent optical networks: Wavelength constraints in routing and signaling. IEEE Commun. Mag., 47: 26-34. DOI: 10.1109/MCOM.2009.5181889

Song, H., B.W. Kim and B. Mukherjee, 2010. Longreach optical access networks: a survey of research challenges, demonstrations and bandwidth assignment mechanisms. IEEE Commun. Surv. Tuts., 12: 112-123. DOI: 10.1109/SURV.2010.020110.00040
Tibuleac, S. and M. Filer, 2010. Transmission impairments in DWDM networks with reconfigurable optical add-drop multiplexers. J. Lightwave Tech., 28: 557-598. DOI: 10.1109/JLT.2009.2037832

Wu, G., T. Zhang, J. Chen, X. Li and C. Qiao, 2011. An index-based parallel scheduler for optical burst switching networks. J. Lightwave Technol., 29: 2766-2773. DOI: 10.1109/JLT.2011.2162485

Zhenga, J. and H.T. Mouftahb, 2009. A survey of dynamic bandwidth allocation algorithms for ethernet passive optical networks. Opt. Switch. Network., $\quad 6$ : 151-162. DOI: 10.1016/j.osn.2009.03.003 